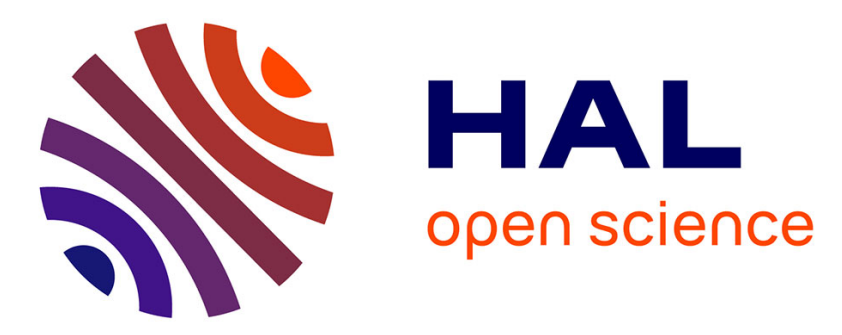

\title{
Qualification and validity of a smeared fractures modeling approach for flow and transport in fractured media
}

\author{
A Fourno, C. Grenier, E. E. Mouche, G Thouvenin, H Benabderrahmane
}

\section{- To cite this version:}

A Fourno, C. Grenier, E. E. Mouche, G Thouvenin, H Benabderrahmane. Qualification and validity of a smeared fractures modeling approach for flow and transport in fractured media. Developments in Water Science 47:319-326, 2002. hal-01738231

\section{HAL Id: hal-01738231 \\ https://hal-ifp.archives-ouvertes.fr/hal-01738231}

Submitted on 20 Mar 2018

HAL is a multi-disciplinary open access archive for the deposit and dissemination of scientific research documents, whether they are published or not. The documents may come from teaching and research institutions in France or abroad, or from public or private research centers.
L'archive ouverte pluridisciplinaire HAL, est destinée au dépôt et à la diffusion de documents scientifiques de niveau recherche, publiés ou non, émanant des établissements d'enseignement et de recherche français ou étrangers, des laboratoires publics ou privés. 


\title{
Qualification and validity of a smeared fractures modeling approach for flow and transport in fractured media
}

\author{
A. Fourno ${ }^{a}$, C. Grenier ${ }^{a}$, E. Mouche ${ }^{a}$, G. Thouvenin ${ }^{a *}$ and H. Benabderrahmane ${ }^{\text {b }}$ \\ ${ }^{a}$ CEA (Commissariat l'Energie Atomique), DEN/DM2S/SFME/MTMS, Centre d'tudes \\ de Saclay, 91191 Gif sur Yvette Cedex, France \\ ${ }^{\mathrm{b}}$ ANDRA (Agence nationale pour la gestion des dchets radioactifs). DS/SHG. \\ Parc de la Croix Blanche. 1-7 rue Jean Monnet. 92298 Chtenay-Malabry, France
}

Modeling flow and transport in a natural fractured medium is a difficult issue due among others to the large contrasts in the medium, its geometrical complexity as well as the strong level of uncertainties for all parameters leading to stochastic modeling.

We present here a contribution to the smeared fracture approach answering the above requisites. It is a continuous representation of the fractured block for a regular discretization mesh, the presence of the fracture network being accounted for by a heterogeneous field of the parameters. Different realization can be easily considered within a Monte Carlo framework addressing geometrical uncertainties while working on coarse mesh allows for reduction of the computer costs.

A smeared fracture method is developed, adapted to flow and transport for a Mixed and Hybrid Finite Element numerical scheme. This approach, developed so far for 2D problems, is evaluated on test cases involving comparisons with simulation performed on the same geometry, but modeled explicitly.

Results show that major transport features are captured by smeared fracture model and allow for a good quantitative restitution of the transfers. This makes this approach an interesting compromise between precision and versality associated to low computer costs in the perspective of Monte Carlo simulations. The limits of the approach for large cinetical contrasts throughout the domain will be further studied in the future.

\section{Introduction}

Modeling transfers in fractured media is a key issue for different fields of application like for instance hydrogeology, petroleum industry, and, as considered here, storage of nuclear wastes. Nevertheless, modeling flow and transport in a natural fractured medium still remains a difficult issue. This is first due to the large geometrical complexity of the fracture network as well as necessity to deal with strong contrasts in the parameter value relative to the fracture zones (flow zones or channels typically corresponding to quick transfer zones) and the matrix blocks (slow transfer zones but associated with large storage capacities). Second, due to the large uncertainties in the localization of the

*Now at CEA, DEN/SAMRA/LGEM, Centre d'tudes de Cadarache, 13108 St Paul lez Durance Cedex 
conductive features, several geometrical configurations are solution to the problem leading to stochastical modeling strategies.

Numerous types of modeling approaches have been developed depending on the connectivity of the fracture network, the modeling scale as compared to the fracturation scale as well as the type of application considered (see e.g. [1]). These approaches typically range from discrete representations of the conducting features (Discrete Fracture Network method) to continuous representations of the fractured block.

We focus here on a continuous representation of the medium involving a modeling region corresponding to the fracturation scale : several conductive features have to be explicitly considered within a fractured block. The fracture network is not explicitly meshed here but the presence of fractures is taken into account by means of continuous but heterogeneous fields (permeability, porosity, head, velocity, concentration). This line is followed by different authors along two main lines : stochastic continuum models $[8,3]$ or as developed here, smeared fracture approach $[7,6,3]$. This type of method presents the following advantages : (i) no dedicated spatial discretization effort is required (we use a basic regular mesh), (ii) simulations can be done on a rough mesh saving computer time. So this kind of approach is very promising for taking heterogeneity of properties as well as uncertainties into account within a Monte Carlo framework for instance. Furthermore, the geometry of the matrix blocks where transfers proceed by diffusion is fully taken into account, contrary to discrete methods.

Nevertheless, this continuous representation of a fracture medium can be considered as an alternative conceptual model or as an approximation to a more classical modeling involving explicit representation of the conductors. For the latter case, it consequently requires solving a homogenization problem at the discretization scale. Conditions on the mesh size have to be stated : it should be chosen sufficiently fine to capture the main geometrical features but coarse enough to save computer time in the simulation. Literature proves that although well tested homogenization approaches exist for permanent flow problems [5,4], homogenizing transitory transport features remains a challenging task.

We propose here a new approach dedicated to Mixed and Hybrid Finite Element numerical scheme [2]. This scheme, implemented in our CAST3M code, shows very good potentials since its simulation performance (e.g. conservation of mass) are particularly good for for highly heterogeneous media. In the following, the smeared approach method developed is described for 2D flow and transport problems. It is applied to a simple 4 fracture geometry for evaluation and qualification of the approach against reference calculations based on classical explicit representation of the geometry. Sensitivity to discretization is studied.

\section{Permanent flow problem}

The equations governing permanent flow are classically, for $q\left(m^{2} / s\right)$ Darcy velocity, $T$ $\left(m^{2} / s\right)$ transmissivity, $h(\mathrm{~m})$ head.

$\vec{q}=-T * g r \overrightarrow{r a d h}$

$$
\operatorname{div}(\vec{q})=0
$$

The first step of the method consists in introducing the geometrical features of the fracture network in the grid : the elements of the regular mesh intersected by a fracture 
are identified and assembled to form a connected tube-like geometry (see figure 1 and 2 for the 4 conductor case considered below).

In a second step, parameter values are associated to these elements (transmissivity, porosity, dispersion tensor ...). These are equivalent properties. The homogenization procedure is conducted at the level of each fracture, assuming no heterogeneity within each conductor. As such it differs from other smeared fracture approaches $[7,6]$ treating the homogenization process at the level of each discretization mesh. Nevertheless our approach allows for an accurate restitution of the total mass flux transiting through the fracture. This is done for each of the fractures in the network. The remaining point consists in choosing a value for intersections : we chose the maximum of the values. This constitutes a deviation to the strict equivalence obtained for single conductors. The quality of the equivalence for the resulting network is then tested against reference calculations.

We sketch out here the homogenization procedure followed for the flow problem (determination of the equivalent transmissivity). As mentioned above, the geometry affected by the procedure remains limited to a one mesh thick tube and as such is only smeared on one mesh. The equivalence of the flux through the reference fracture and the equivalent conductor is easy to write based on the numerical scheme considered. For Mixed and Hybrid Finite Element, conservation of the quantities is guaranteed for each mesh by the introduction of four unknowns (traces affected to the center of the element edges in addition to the classical unknowns corresponding to the center of the elements). These allow for jointed estimation of the fluxes (gradients) as well as the values of the unknowns within the mesh. Considering permanent flow limited to the fractures (matrix blocks are assumed no flow zones), heads at upstream and downsteam locations are related through a 1D tube involving two types of elements : a first type propagates flow from one side to the opposite and the other from one side to the adjacent (a turn). For the discretization adopted, the relation between flow and head difference at the inlet and outlet can be easily obtained. For a given angle, the number of each element involved ( $X$ for the first type and $Y$ for the second type) put in series leads to the equivalent transmissivity :

$T_{s f}=\frac{(3 X+2 Y) e}{3 L} T_{r e f}$,

where $e, L$ and $T_{f}$ are respectively the thickness, the length and the permeability of the fracture. The subscrips $s f$ and $r e f$ are respectively related to smeared fracture equivalent property and reference values for explicit modeling.

This equivalence is exact for a single conductor. Nevertheless, dealing with several fractures introduces deviances from this due to the treatment of the intersections (maximal transmissivity is chosen). The quality of the procedure is evaluated on a test case consisting of a four fracture geometry as shown on figure 1. The boundary conditions applied are shown on figure 3 providing the reference head field as well as the boundary conditions. The flow is globally from bottom to top right. Reference calculations are conducted on the explicitly meshed geometry for the same Mixed Hybrid Finite Element scheme and for the discretization represented on figure 1. Flow and head fields for the reference case and the smeared fracture system (see geometry figure 2) are simulated. Results of the comparison show that both simulations are qualitatively similar. The total flux through the system as well as the local flux in the major conductors typically differ by one percent. 
Nevertheless, detailed examination of the local fluxes within minor conducting features shows that errors can span up to several percents or tenth of percents. Sensitivity analysis to the size of the mesh was conducted. Results show that multiplying the size of the mesh by two or dividing it by two does not lead to major quantitative differences in the values of the flux. A lower limit nevertheless appears for coarser meshing and can be expressed as : each matrix block (or similarly fracture section) needs be discretized into several meshes. If this is not done, the block is skipped and turned into an intersection.

Further evaluation of the problem are considered for other reference geometries and parameter values. Nevertheless as a preliminary conclusion, the approach appears robust, qualitatively efficient (percent error scale) and saves computer time. Nevertheless, the larger relative errors in the simulation of local flow within minor conductors will play a larger role for the transport problem as shown below.

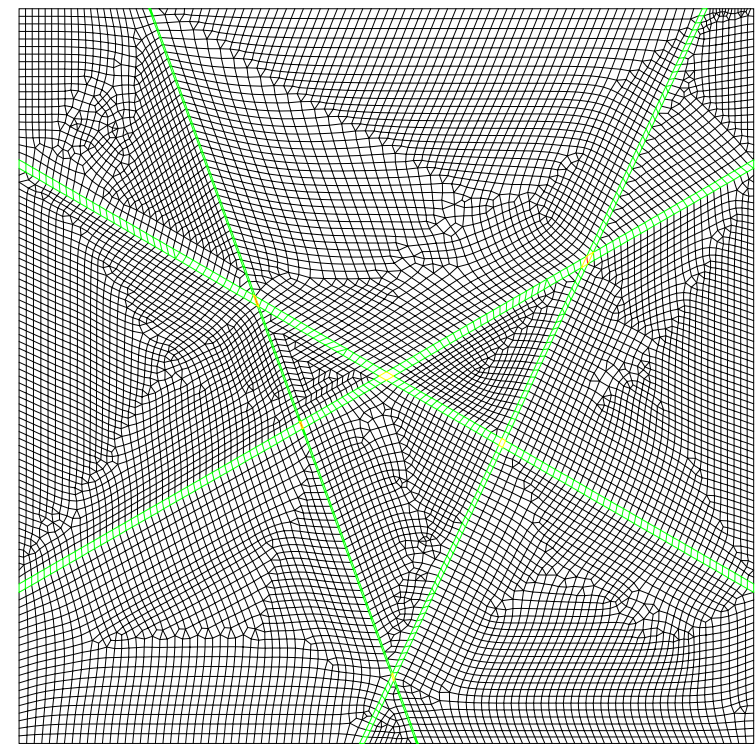

Figure 1. Four fracture system : discretization for the reference calculation.



Figure 2. Four fracture system (meshes extracted, smeared fracture approach). Initial concentration is located in Zone 0.

\section{Transport problem}

Two distinct modeling strategies were implemented in the smeared fracture approach : Lagrangian and Eulerian.

The Lagrangian approach is particularly adapted to advective transport or high Peclet cases. This method has been implemented, showed computer efficient and was tested with success against reference simulations. It requires theoretical derivation of equivalent porosity as well as a slight modification of the particle tracking procedure available within CAST3M code. Nevertheless it can't be easily extended to account for diffusion phenom- 
ena and in particular matrix diffusion due to the strong contrasts in the velocity field among the smeared fracture meshes : in short, for a second type mesh, flow entering a side of the mesh and exiting the contiguous side, the transfer times vary from nearly zero to very large values depending on the initial position of the particle entering the element. So, Lagrangian approach remains limited here to purely advective transfers within the fracture network and is not further developped.

The Eulerian approach presents larger potentials to model matrix diffusion and is appropriate for lower Peclet values. Our purpose is to develop the method in order to take matrix diffusion into account in a near future, for the actual matrix blocks geometry. We present here the part of the study related to transport within the fracture network only.

The transfer of mass under advective and dispersive (diffusive) mechanisms can be classically represented by the following equation :

$\omega \frac{\partial C}{\partial t}=\operatorname{div}(\overline{\bar{D}} g r \vec{a} d C-C \vec{q})$

where $C$ stands for concentration $\left(\mathrm{kg} / \mathrm{m}^{3}\right), \overline{\bar{D}}=\omega * D_{p}+\alpha * q$ is the dispersion tensor, $\omega$ porosity, $D_{p}$ pore diffusion coefficient $(\mathrm{m} / \mathrm{s}), \alpha$ dispersivity coefficient $(\mathrm{m})$, and $\vec{q}$ Darcy velocity $\left(m^{2} / s\right)$.

Smeared fracture approach for transport relies upon the permanent flow modeling discretization and parameter estimation presented previously. The transport phase requires estimation of further equivalent properties associated to the mesh : porosity and dispersion tensor. The equivalent porosity is estimated like for a Lagrangian approach : transition times can be easily calculated for both type of elements encountered on the smeared fracture geometry. Similarly to the derivation of the equivalent transmissivity $X$ elements of the first type and $Y$ of the second type are encountered. The final transition time through the system adds all the transfer times for all elements met on the path. The (strictly) equivalent porosity $\left(\omega_{s f}\right)$ is for $\Delta$ the spatial discretization step :

$\omega_{s f}=\frac{L e}{(X+Y) \Delta^{2}}$

The equivalent dispersion tensor is here derived as a scalar value based on the same considerations as for the equivalent transmissivity. The dispersive diffusive part of the transport equation is analogous to the flow equation leading to the following final result for a single conductor :

$D_{s f}=\frac{(3 X+2 Y) e}{3 L} D_{r e f}, \quad \quad \alpha_{s f}=\frac{(3 X+2 Y)}{3} \frac{\Delta}{L} \alpha_{r e f}$

For a fracture network, the same procedure is applied for each conductor, the value affected to the intersection is the maximum of all met in this mesh. The type of transfer at the fracture intersections is full mixing.

The smeared fracture approach for Eulerian transport within a fracture network was tested on the 4 conductor geometry (see figure 2) comparing smeared fracture results to reference calculations obtained with the explicit geometry (see figure 1). The parameter values associated are provided on table 1 . The flow field corresponds to the previous 
case : mainly from bottom to top of the domain. The initial transport conditions are : unity concentration in one mesh of the domain. Several release positions were studied corresponding to the different zones mentioned on figure 2. We focus here on the Zone 0 case, located upstream at the intersection of two main fractures (numbered 3 and 4). Globally, the plume first migrates within both conductors at different velocities, separates at following intersections and reaches outlets at the top right of the domain following different paths. This leads to dispersion process of the plume through the network. The quantity measured are breakthrough curves at the limits of the domain, concentration fields at different times, temporal evolution of the masses in the fractures and fracture sections. A picture of the concentration field at time $7.810^{4} \mathrm{~s}$ is provided on figure 5 for the smeared fracture simulation. Different levels of discretization were considered including the figure 2 case as well as a twice finer and twice coarser grid.

Results show overall good qualitative as well as quantitative results. Whatever the initial position of the plume, peak arrival time is met with errors close to $5 \%$ whereas peak maximum more largely differs (10 to $20 \%$ depending on the discretization level). Breakthrough curves for zone 0 release are provided on figure 4 including the reference case as well as the smeared fracture simulation for the 3 discretization levels mentioned. For this location, the smeared fracture simulations are less dispersive than the reference calculations. Refined study of the case shows that this difference is mainly due to discrepancies in cinetical transfer patterns situated in short fracture sections having low velocity (here in particular section of fracture 2 between fracture 1 and 3). As mentioned before, larger relative errors for the flow field are located in sections of the system involving low flow rates. In addition to this, time discretization chosen corresponds to the quicker processes leading to local loss of precision in slow velocity zones. These difficulties seem to be intrinsic drawbacks of the approach. They are for instance poorly improved by refined discretization. One should bear in mind that the problem is simulated involving a given single spatial and temporal discretization level. The simulation time is typically improved by a factor larger than 2 as compared with reference calculation.

\section{Conclusions and perspectives}

Although further work is still required to test the approach for different configurations (geometry and parameters values), results already appear very satisfactory. The main features of flow and transport are indeed captured by the smeared fracture method. As such, smeared fracture approach appears as a very interesting compromise between precision of the results, simplicity of the implementation of the method, flexibility dealing with different fracture geometries within a Monte Carlo approach, lower computer costs. Sensitivity to discretization level is rather limited provided that each unit of the system is modeled over several meshes.

We are currently introducing matrix diffusion within the Eulerian approach. A major advantage of the approach consists in dealing with the actual geometry of the matrix blocks contrary to classical 1D orthogonal diffusion models. Preliminary results are promising. Nevertheless one of the major drawback of the method is certainly that since discretization involves constant size of the mesh, large cinetical discrepancies among the modeled domain might lead to loss of precisions in the results. The method provides with 


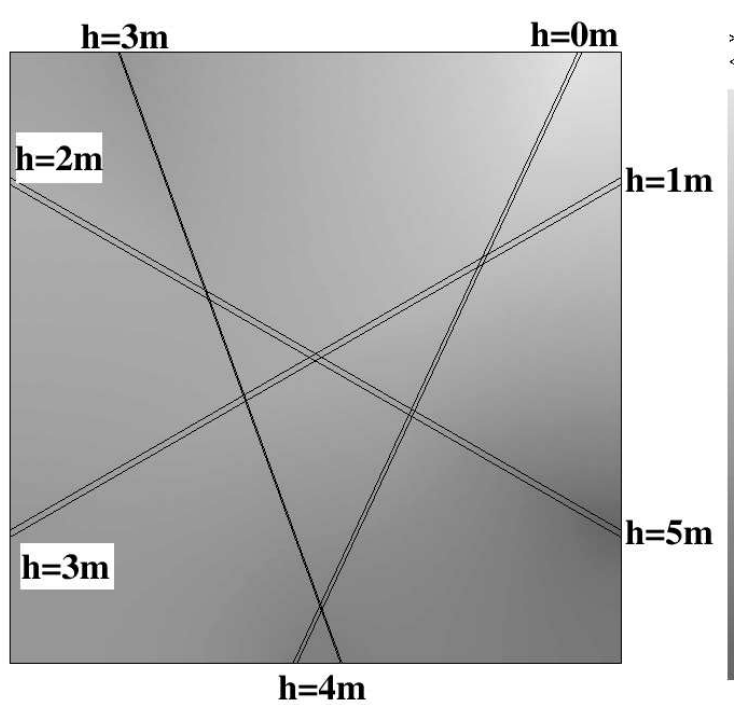

Figure 3. Boundary conditions for flow problem, head field $(\mathrm{m})$.

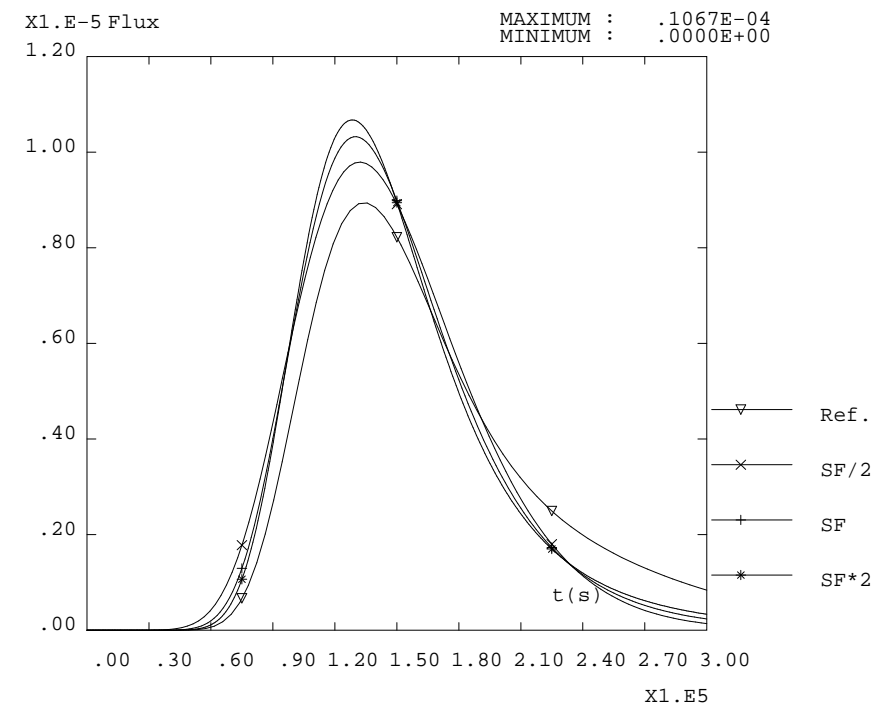

Figure 4. Output flux. Reference vs. smeared fracture (3 discretization levels: original, twice coarser $* 2$ or refined $/ 2$ )

global features obtained for a given single spatial and temporal discretization level and as such does not necessarily captures all the effects present at other scales. The limits of the approach will be further characterized in the future.

\section{REFERENCES}

1. J. Bear, C.-F. Tsang, G. de Marsily (ed.). Flow and contaminant transport in fractured rock. Academic Press, 1993.

2. F. Dabbene. Mixed Hybrid Finite Elements for transport of pollutants by undergound water. Proc. 10th conference on finite elements in fluids, Tucson, Arizona, 1998.

3. J. Gomez-Hernandez, H. Henricks, Franssen, A. Sahuquillo, J. Capilla. Calibration of 3D transient groundwater flow models in fractured rocks. Proc. conf. ModelCARE'99, Zurich, Switzerland, 1999.

4. G. de Marsily, Ph. Renard. Calculating equivalent permeability : a review. Advances in Water Resources V. 20, N. 5-6, P. 253-278, 1997.

5. X. Sanchez-Villa, J. P. Girardi, J. Carrera. A synthesis of approaches to upscaling of hydraulic conductivities. Water Resources Research V. 31, N. 4, P. 867-882, 1995.

6. U. Svensson. A continuum representation of fracture networks. Part I : Method and basic test cases. Journal of Hydrology 250, pp. 170-186, 2001.

7. Y. Tanaka, K. Minyakawa, T. Igarashi, Y. Shigeno. Application of 3D smeared fracture model to the hydraulic impact of the Aspo tunnel. SKB Report. ICR 96-07, 1996.

8. Y. W. Tsang, C. F. Tsang, F. V. Hale, B. Deverstorp, Tracer transport in a stochastic continuum model of fractured media. Wat. Res. Res., V. 32, N. 10, P. 3077-3092, 1996. 
Table 1

\begin{tabular}{|c|c|c|c|c|}
\hline Parameters & Fracture 1 & Fracture 2 & Fracture 3 & Fracture 4 \\
\hline Fracture apertures $(m)$ & $e=0.5$ & $e=0.2$ & $e=0.3$ & $e=0.1$ \\
\hline Transmissivity $\left(m^{2} . s^{-1}\right)$ & $10^{-2}$ & $10^{-3}$ & $5.10^{-3}$ & $3.10^{-3}$ \\
\hline Angle $\left(^{o}\right)$ & 30 & 150 & 65 & 110 \\
\hline Length $(m)$ & 57.7 & 57.5 & 55. & 52.5 \\
\hline
\end{tabular}

Parameter set considered. Porosity equals 1 for all fractures

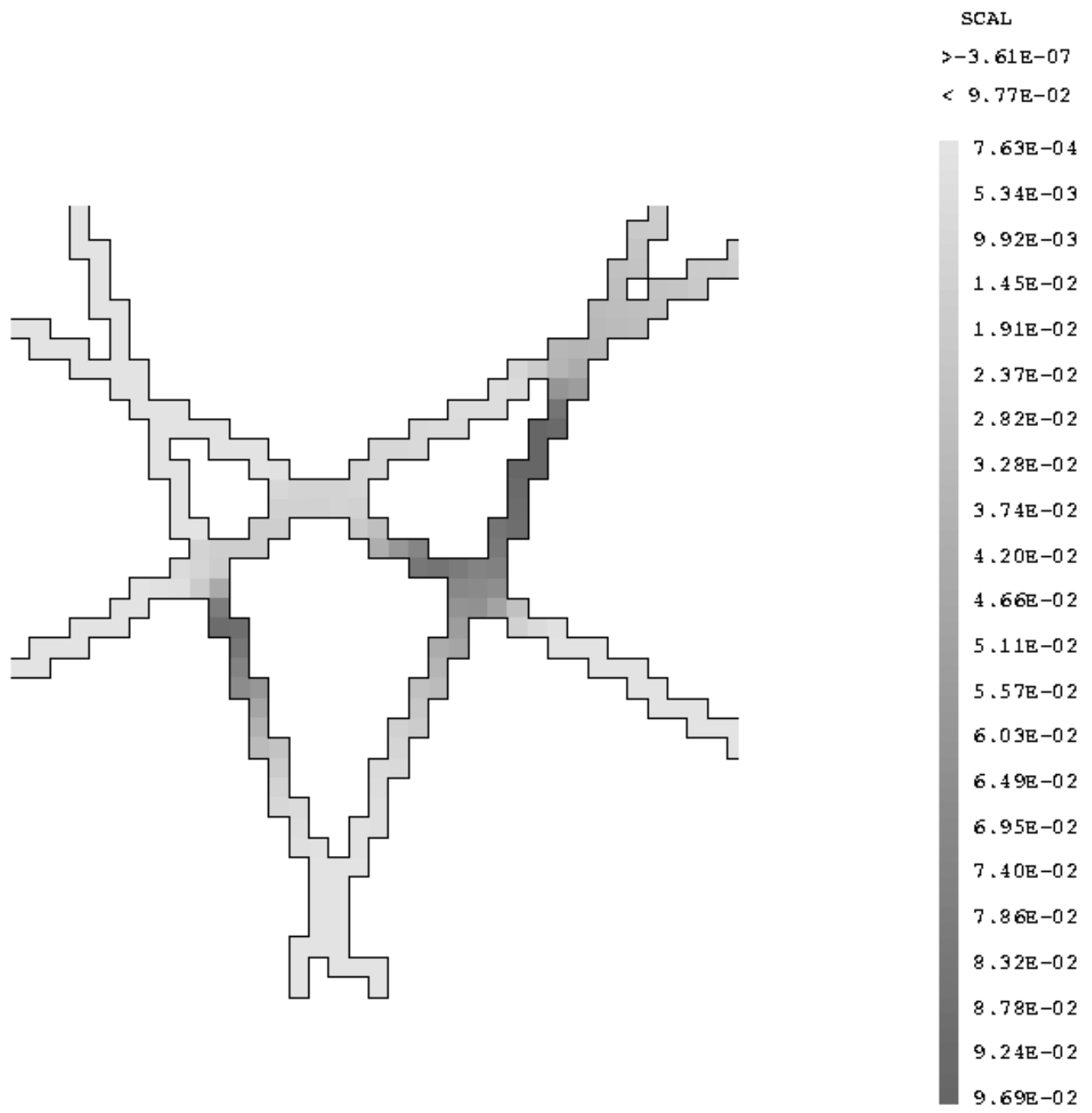

Figure 5. Concentration field for time $7.810^{4} \mathrm{~s}$ : smeared fracture simulation. The same features appear on the reference calculation for the same time (figure not provided here). Initial unit concentration was originally located in zone 0. 